Relations industrielles

Industrial Relations

\title{
Labor Law ; par Nicholas S. Falcone ; John Wiley and Sons, Inc., New York, N.Y., 1962, 502 pp.
}

Jean-Réal Cardin

Volume 17, numéro 4, octobre 1962

URI : https://id.erudit.org/iderudit/1021495ar

DOI : https://doi.org/10.7202/1021495ar

Aller au sommaire du numéro

Éditeur(s)

Département des relations industrielles de l'Université Laval

ISSN

0034-379X (imprimé)

1703-8138 (numérique)

Découvrir la revue

Citer ce compte rendu

Cardin, J.-R. (1962). Compte rendu de [Labor Law ; par Nicholas S. Falcone ;

John Wiley and Sons, Inc., New York, N.Y., 1962, 502 pp.] Relations industrielles /

Industrial Relations, 17(4), 508-509. https://doi.org/10.7202/1021495ar

Tous droits réservés (C Département des relations industrielles de l’Université Laval, 1963
Ce document est protégé par la loi sur le droit d'auteur. L'utilisation des services d'Érudit (y compris la reproduction) est assujettie à sa politique d'utilisation que vous pouvez consulter en ligne.

https://apropos.erudit.org/fr/usagers/politique-dutilisation/ 
books », édité conjointement par l'université anglaise de Cambridge et l'université de Chicago. Le but de cette collection, c'est de fournir à des lecteurs non-initiés une idée des principes que les économistes utilisent dans l'analyse des problèmes économiques contemporains. L'intérêt de cette série est donc centré sur le fait qu'elle s'adresse à des lecteurs ordinaires et qu'elle traite de problèmes courants.

Plus précisément, le livre du professeur Rees comporte principalement une discussion du rôle joué par les unions dans l'élaboration de la structure des salaires et de l'effet des changements dans cette dernière sur les prix, l'emploi, la productivité et la part des salariés dans le revenu national. Il complète de cette façon les livres connus des professeurs Ross et Dunlop et incorpore l'essentiel de la littérature parue à cet égard depuis la publication des livres de ces derniers.

Bien que ce soit l'intention de l'auteur de traiter surtout de l'aspect économique des unions ouvrières, le livre contient certains chapitres où l'on trouve l'essentiel de l'aspect institutionnel des unions. Cela permet de situer le rôle économique des unions dans son cadre institutionnel.

A cause de la qualité des lecteurs auxquels il s'adresse avant tout, l'auteur n'utilise pas les techniques d'analyse habituelles aux économistes : on ne trouvera dans le livre ni graphique, ni relations mathématiques qui risqueraient de rebuter les non-initiés. Cette absence cependant n'enlève rien à la valeur scientifique du livre. La rigueur de la démonstration est toujours apparente et l'absence d'un appareil technique ne conduit pas au verbiage. Au contraire, on remarquera la concision avec laquelle les problèmes sont discutés.

L'auteur a su éviter l'allure théorique et artificielle qui est le propre de certaines analyses économiques en illustrant ses propres références à des situations bien concrètes.

Le livre de M. Rees est certainement la présentation systématique la plus sûre, la plus complète et la plus à date du rôle économique des unions ouvrières. C'est pourquoi nous le recommandons fortement à l'attention de tous ceux qui se réjouissent ou qui s'inquiètent du rôle de cette dernière dans notre so. ciété.

\section{Jacques St-Laurent}

Labor Law; par Nicholas S. Falcone; John Wiley and Sons, Inc., New York, N.Y., 1962, 502 pp.

Le présent ouvrage se situe dans la lignée bibliographique des ouvrages consacrés au droit du travail chez nos voisins du Sud. Nous connaissions déjà les éditions de l'ouvrage de Charles $\mathbf{O}$. Gregory «Labor and the Law », le répertoire de Benjamin Werne «The Law of Industrial Relations », le traité de Glenn Miller «American Labor and the Government » ainsi que certains autres ouvrages de moindre envergure, sans compter l'immense littérature à caractère juridique ou para-juridique que les Américains ont produite depuis un certain nombre d'années en rapport avec les relations industrielles chez eux.

Une telle évolution s'explique assez bien si l'on songe au caractère fortement légaliste du système américain de relations industrielles, à l'importance et à l'universalité qu'ont acquises les règles juridiques de ce système au fur et à mesure que les pouvoirs publics sont intervenus dans ce champ d'activités au moyen de lois-cadres, de statuts particuliers, de réglementations de toutes sortes, et que les tribunaux aussi bien judiciaires qu'administratifs ont été appelés à interpréter et à appliquer une telle masse de législation.

L'ouvrage de Falcone suit une méthodologie que nous appellerions «traditionnelle », en matière de traités de droit du travail américains; il se présente toutefois selon un ordre chronologique qui permet à l'auteur de situer les grandes étapes de la législature ouvrière, d'en faire ressortir les principales caractéristiques, d'en montrer l'évolution en fonction des grands textes législatifs et des principales décisions judiciaires qui en ont informé la marche au cours des années.

Comme plusieurs autres ouvrages semblables, le présent traité contient en appendice les textes mêmes des principales lois ouvrières américaines adoptées depuis quarante ans et qui ont servi à constituer l'armature du système actuel de relations industrielles aux Etats-Unis. 
L'auteur laisse délibérément de côté les lois de protection et de sécurité sociale, telles les lois de salaires minimum, de manufactures, d'accidents du travail, etc., pour se concentrer plus spécifiquement sur le corps de droit commun, statutaire et administratif régissant les relations collectives de travail et en suivre l'évolution des débuts jusqu'à nos jours.

Encore une fois, s'il ne s'agit pas d'un ouvrage historique, il reflète tout de même le désir de l'auteur de présenter au lecteur l'arrière-plan indispensable à une bonne compréhension des problèmes présents. Chaque texte de loi, ainsi que les principales décisions judiciaires qui $y$ sont attachées, sont étudiées dans l'ouvrage de Falcone. Nul doute que ceux qui s'intéressent au droit du travail, étudiants comme professeurs ou praticiens du droit, trouveront ce procédé très utile.

On peut cependant faire remarquer au sujet du livre de Falcone, comme d'ailleurs au sujet de tous les autres du même genre, que les politiques gouvernementales en matières de relations industrielles évoluant constamment, les décisions, surtout au niveau des cours inférieurs, étant loin d'être constantes, et qu'enfin les décisions du Bureau national des relations ouvrières venant assez souvent en conflit avec celles des tribunaux fédéraux, le lecteur, s'il veut acquérir une connaissance exacte du droit ouvrier de nos voisins, ne doit pas se limiter à la lecture d'un seul traité, mais s'efforcer de comparer à l'aide d'autres ouvrages de référence.

L'ouvrage est divisé en deux parties. $\mathrm{La}$ première traite de l'évolution du droit ouvrier en Grande-Bretagne; la seconde couvre d'une façon complète lévolution de ce même droit aux EtatsUnis; ce que ne font pas, d'une façon aussi systématique, certains ouvrages similaires.

Y est aussi présentée, en fonction de chaque période et de chaque loi majeure, une jurisprudence expliquée qui nous aide à saisir mieux la substance du droit américain du travail. Plusieurs chapitres s'occupent plus particulièrement des règles régissant la grève, le piquet et le boycottage à l'occasion des conflits du travail. Un dernier chapitre traite de l'arbitrage tel qu'il se pratique aux Etats-Unis, ainsi que des autres procédures de règlement des conflits, telles que conciliation et médiation. Ce livre constitue un précieux répertoire, ainsi qu'un traité analytique des plus utiles au juriste et à l'homme de relations industrielles qui s'intéressent au droit comparé du travail.

\section{J.-R. Cardin}

Le problème des Relations collectives du Travail en Belgique: Edité par la Confédération des Syndicats chrétiens de Belgique : XXe Congrès, Bruxelles, 19-21 octobre 1962 ; Etudes économiques et sociales XI, 1962 ; Editions de la C.S.C., 127, rue de la Loi, Bruxelles, 1962, $108 \mathrm{pp}$.

Voici une petite plaquette sans prétention que nous livre la Confédération des Syndicats chrétiens de Belgique. En dépit de ses dimensions réduites, elle nous offre une image singulièrement complète et dépouillée du système des relations industrielles belge, tel qu'il évolue présentement en ce pays. On retrouve dans ce compte-rendu clair, succinct, bien ordonné et précis, les qualités traditionnelles auxquelles les ouvrages européens sur le sujet nous ont habitués.

Evidemment, le traitement apporté ici à la matière est élémentaire en tous points ; il n'entre dans aucune complexité qu'un ouvrage plus fouillé devrait nécessairement aborder. Il reste cependant, qu'une fois ces précisions établies, et que la fin de vulgarisation qu'il se propose est admise par le lecteur, ce dernier ne peut faire autrement qu'être impressionné par la facture méthodologique qu'un tel précis utilise.

A plusieurs titres, ce petit ouvrage nous rappelle ceux du Bureau international du Travail qui composent la série consacrée à l'étude de la situation syndicale dans les pays membres et dont quelques-uns nous sont déjà parvenus. Comme ces derniers, il utilise d'abord les cadres institutionnels pour exposer dans leurs dimensions essentielles les principaux problèmes de relations du travail tels qu'ils se présentent dans le pays étudié, en l'occurrence la Belgique. Profitons de l'occasion pour déplorer une fois de plus l'absence d'ouvrages semblables, pour fins d'éducation populaire, relativement à notre propre système de relations industrielles ici au Québec.

Après une brève introduction retraçant à grands traits les étapes de l'his- 